\title{
COSMOS: HUBBLE SPACE TELESCOPE OBSERVATIONS ${ }^{1}$
}

\author{
N. Scoville, ${ }^{2,3}$ R. G. Abraham, ${ }^{4}$ H. Aussel, ${ }^{5,6}$ J. E. Barnes, ${ }^{5}$ A. Benson, ${ }^{2}$ A. W. Blain, ${ }^{2}$ D. Calzetti, ${ }^{7}$ \\ A. Comastri, ${ }^{8}$ P. Capak, ${ }^{2}$ C. Carilli, ${ }^{9}$ J. E. Carlstrom, ${ }^{10}$ C. M. Carollo, ${ }^{11}$ J. Colbert, ${ }^{12}$ E. Daddi, ${ }^{13}$ \\ R. S. Ellis, ${ }^{2}$ M. Elvis, ${ }^{14}$ S. P. Ewald, ${ }^{2}$ M. Fall, ${ }^{7}$ A. Franceschini, ${ }^{15}$ M. Giavalisco, ${ }^{7}$ W. Green, ${ }^{2}$ \\ R. E. Griffiths, ${ }^{16}$ L. Guzzo, ${ }^{17}$ G. Hasinger, ${ }^{18}$ C. Impey, ${ }^{19}$ J.-P. Kneib, ${ }^{20}{ }^{2}$ J. Koda, ${ }^{2}$ A. KoeKemoer, ${ }^{7}$ \\ O. Lefevre, ${ }^{20}$ S. Lilly, ${ }^{11}$ C. T. Liu, ${ }^{21}$ H. J. McCracken, ${ }^{22,23}$ R. Massey, ${ }^{2}$ Y. Mellier, ${ }^{22}$ \\ S. Miyazaki, ${ }^{24}$ B. Mobasher, ${ }^{7}$ J. Mould, ${ }^{25}$ C. Norman, ${ }^{26}$ A. Refregier, ${ }^{6}$ A. Renzini, ${ }^{15,27}$ \\ J. Rhodes, ${ }^{2,28}$ M. Rich, ${ }^{29}$ D. B. SAnders, ${ }^{5}$ D. Schiminovich, ${ }^{30}$ E. Schinnerer, ${ }^{31}$ \\ M. Scodeggio, ${ }^{32}$ K. Sheth, ${ }^{2,12}$ P. L. Shopbell, ${ }^{2}$ Y. Taniguchi, ${ }^{33}$ \\ N. D. Tyson, ${ }^{34}$ C. M. URry, ${ }^{35}$ L. VAN WaerbeKe, ${ }^{36}$ \\ P. Vettolani, ${ }^{8}$ S. D. M. White, ${ }^{37}$ and L. Yan ${ }^{12}$ \\ Received 2006 April 24; accepted 2006 June 28
}

\begin{abstract}
The Cosmic Evolution Survey (COSMOS) was initiated with an extensive allocation (590 orbits in Cycles 12-13) using the Hubble Space Telescope (HST) for high-resolution imaging. Here we review the characteristics of the HST imaging with the Advanced Camera for Surveys (ACS) and parallel observations with NICMOS and WFPC2. A square field $\left(1.8 \mathrm{deg}^{2}\right)$ has been imaged with single-orbit ACS I-band F814W exposures with 50\% completeness for sources $0.5^{\prime \prime}$ in diameter at $I_{\mathrm{AB}}=26.0 \mathrm{mag}$. The ACS is a key part of the COSMOS survey, providing very high sensitivity and high-resolution ( $0.09^{\prime \prime}$ FWHM and $0.05^{\prime \prime}$ pixels) imaging and detecting a million objects. These images yield resolved morphologies for several hundred thousand galaxies. The small HST PSF also provides greatly enhanced sensitivity for weak-lensing investigations of the dark matter distribution.
\end{abstract}

Subject headings: cosmology: observations — dark matter — galaxies: evolution — galaxies: formation — large-scale structure of universe - surveys

\footnotetext{
1 Based on observations with the NASA/ESA Hubble Space Telescope, obtained at the Space Telescope Science Institute, which is operated by Association of Universities for Research in Astronomy, Inc., under NASA contract NAS5-26555.

2 California Institute of Technology, MC 105-24, 1200 East California Boulevard, Pasadena, CA 91125.

3 Visiting Astronomer, University of Hawaii, 2680 Woodlawn Drive, Honolulu, HI 96822.

4 Department of Astronomy and Astrophysics, University of Toronto, 60 St. George Street, Room 1403, Toronto, ON M5S 3H8, Canada.

5 Institute for Astronomy, 2680 Woodlawn Drive, University of Hawaii, Honolulu, HI 96822.

6 Service d'Astrophysique, CEA/Saclay, F-91191 Gif-sur-Yvette, France.

7 Space Telescope Science Institute, 3700 San Martin Drive, Baltimore, MD 21218.

8 INAF-Osservatorio Astronomico di Bologna, via Ranzani 1, I-40127 Bologna, Italy.

9 National Radio Astronomy Observatory, P.O. Box O, Socorro, NM 87801-0387.

10 Department of Physics, University of Chicago, 5640 South Ellis Avenue, Chicago, IL 60637.

11 Department of Physics, Eidgenössische Technische Hochschule Zurich, CH-8093 Zurich, Switzerland.

12 Spitzer Science Center, California Institute of Technology, Pasadena, CA 91125.

13 National Optical Astronomy Observatory, P.O. Box 26732, Tucson, AZ 85726; currently at Laboratoire AIM, CEA/DSM - CNRS - Université Paris Diderot, DAPNIA/SAp, Orme des Merisiers, F-91191 Gif-sur-Yvette, France.

14 Harvard-Smithsonian Center for Astrophysics, 60 Garden Street, Cambridge, MA 02138.

15 Dipartimento di Astronomia, Università di Padova, vicolo dell'Osservatorio 2, I-35122 Padua, Italy.

16 Department of Physics, Carnegie Mellon University, 5000 Forbes Avenue, Pittsburgh, PA 15213.

17 Osservatorio Astronomico di Brera, via Brera, I-20121 Milan, Italy.

18 Max-Planck-Institut für Extraterrestrische Physik, D-85478 Garching, Germany.

19 Steward Observatory, University of Arizona, 933 North Cherry Avenue, Tucson, AZ 85721.

20 Laboratoire d'Astrophysique de Marseille, BP 8, Traverse du Siphon, F-13376 Marseille Cedex 12, France.

21 Astrophysical Observatory, City University of New York, College of Staten Island, 2800 Victory Boulevard, Staten Island, NY 10314.

22 Institut d'Astrophysique de Paris, UMR7095 CNRS, Université Pierre et Marie Curie, 98 bis Boulevard Arago, F-75014 Paris, France.

23 Observatoire de Paris, LERMA, 61 Avenue de l'Observatoire, F-75014 Paris, France.

24 Subaru Telescope, National Astronomical Observatory of Japan, 650 North Aohoku Place, Hilo, HI 96720.

25 National Optical Astronomy Observatory, P.O. Box 26732, Tucson, AZ 85726.

26 Department of Physics and Astronomy, Johns Hopkins University, Homewood Campus, Baltimore, MD 21218.

27 European Southern Observatory, Karl-Schwarzschild-Strasse 2, D-85748 Garching, Germany.

28 Jet Propulsion Laboratory, Pasadena, CA 91109.

29 Department of Physics and Astronomy, University of California, Los Angeles, CA 90095.

30 Department of Astronomy, Columbia University, MC 2457, 550 West 120th Street, New York, NY 10027.

31 Max-Planck-Institut für Astronomie, Königstuhl 17, D-69117 Heidelberg, Germany.

32 Instituto di Astrofisica Spaziale e Fisica Cosmica, CNR, via Bassini 15, I-20133 Milano, Italy.

33 Physics Department, Graduate School of Science, Ehime University, 2-5 Bunkyo-cho, Matsuyama 790-8577, Japan.

34 American Museum of Natural History, Central Park West at 79th Street, New York, NY 10024.

35 Department of Astronomy, Yale University, P.O. Box 208101, New Haven, CT 06520-8101.

36 Institut d'Astrophysique de Paris, 98 bis Boulevard Arago, F-75014 Paris, France.

37 Max-Planck-Institut für Astrophysik, D-85748 Garching bei München, Germany.
} 


\section{INTRODUCTION}

Sensitive, high-resolution imaging is a critical component of all cosmological evolution studies, especially for surveys probing the evolution of luminous galaxies at redshift $z>0.5$, when most galaxy assembly and evolution occurred. This approach was initiated in the Hubble Space Telescope (HST) Treasury surveys: first, the Hubble Deep Fields (HDFs; Williams et al. 1996, 2000), which imaged a $5 \operatorname{arcmin}^{2}$ area, followed by GOODS (Giavalisco et al. 2004), which covered a larger area (360 $\left.\operatorname{arcmin}^{2}\right)$, GEMS (Rix et al. 2004), which was still more extensive $\left(800 \mathrm{arcmin}^{2}\right)$ but at shallower depth, and, most recently, the Ultra Deep Field (UDF) survey (Beckwith et al. 2006), which was extremely deep but covered only $11 \mathrm{arcmin}^{2}$. The Cosmic Evolution Survey (COSMOS), with a $2 \mathrm{deg}^{2}$ field, is the first HST survey specifically designed to thoroughly probe the evolution of galaxies, AGNs, and dark matter in the context of their cosmic environment (i.e., large-scale structure [LSS]). COSMOS samples all relevant scales of LSS, up to $\sim 50-100 h_{70}^{-1} \mathrm{Mpc}$ at all $z>0.5$. The area of COSMOS was designed to sample the full dynamic range of large-scale structures from voids to very massive clusters. (HST ACS coverage of the DEEP Groth strip spans $\sim 10^{\prime} \times 70^{\prime}$ [Davis et al. 2007], similar to GEMS, but the elongated geometry is not optimum for sampling the larger structures.) High-resolution imaging with HST enables accurate determination of galaxy morphologies and multiplicities. The HST imaging also provides significantly improved weak-lensing analysis to probe the dark matter distribution of the LSS.

COSMOS is the largest HST survey ever undertaken, imaging an equatorial field with single-orbit $I$-band exposures to a depth $I_{\mathrm{AB}} \simeq 28$ mag ( $5 \sigma$ on an optimally extracted point source) and $50 \%$ completeness for sources $0.5^{\prime \prime}$ in diameter at $I_{\mathrm{AB}}=26.0 \mathrm{mag}$. With this area coverage and sensitivity, COSMOS detects $\simeq 2 \times$ $10^{6}$ objects and samples a volume in the high-redshift universe approaching that sampled locally by the Sloan Digital Sky Survey (SDSS). In this article we describe the observations which make up the HST component of the COSMOS survey: the primary ACS imaging and the parallel NICMOS and WFPC2 imaging. A detailed description of the HST data processing is provided in Koekemoer et al. (2007).

\section{HST OBSERVATIONS}

The original HST Cycle 12 COSMOS proposal had two major components for imaging with the Advanced Camera for Surveys: (1) a complete mosaic of $2 \mathrm{deg}^{2}$ in the F814W ( $I$-band) filter for morphological information, and (2) a similar mosaic in $\mathrm{F} 475 \mathrm{~W}$ ( $g$ band) to provide resolved color imaging for studies of stellar populations and dust obscuration. Although 640 orbits were allocated for the $I$-band imaging, 50 orbits were specified to enable searches for Type Ia supernovae (SNe Ia). Following discussions with the principal investigators for the supernova programs (A. Reiss and S. Perlmutter), the COSMOS team decided that the SN Ia science was not easily served within the COSMOS survey strategy, and the 50 orbits were given over to follow-up exposures in the GOODS-S survey field. Thus, the COSMOS HST survey (590 orbits total) comprises 270 and 320 orbits allocated within HST Cycles 12 and 13 (fall 2003 to spring 2005), respectively.

\subsection{Field Selection}

Multiwavelength imaging and optical spectroscopy are central to the ability of the COSMOS survey to probe the evolution of stellar populations, star formation, galaxies, and AGNs. The enormous investments in observing time required to cover a $2 \mathrm{deg}^{2}$ field rule against having separate northern and southern hemisphere fields, as in earlier, smaller surveys. Thus, an initial prerequisite for COSMOS was that the field be accessible to telescopes in both hemispheres and especially all unique facilities. This precluded using COSMOS to extend the area of earlier survey fields at high northern or southern declination (such as HDF-N/S, GOODS/CDF-N/S, the Lockman Hole, and the Groth strip). An equatorial field is required to enable access by all existing $8-10 \mathrm{~m}$ optical telescopes (and future larger telescopes) and the unique radio facilities (the [E]VLA in the north and ALMA in the south). ALMA is likely to become a "required" facility for studies of early-universe galaxy evolution; at the same time, high-sensitivity VLA radio imaging is critical, and the instrument is unique in terms of sensitivity (with a factor of 3-10 improvement for the EVLA).

The original field proposed for the COSMOS survey was the VIRMOS-VLT Deep Survey (VVDS)/XMM-Newton Deep field centered at R.A. $=2^{\mathrm{h}} 26^{\mathrm{m}}$, decl. $=-4^{\circ} 30^{\prime}(\mathrm{J} 2000.0)$, which was scheduled for extensive optical spectroscopy with the VLT VIMOS spectrograph. However, during the phase two preparation for the COSMOS HST survey in Cycle 12 it became apparent that HST was very overcommitted at R.A. $\sim 2^{\mathrm{h}}$ due to the Ultra Deep Field (UDF) Directors Discretionary Time project in Cycle 12; we were therefore requested by both the STScI and ESO directors to consider shifting the COSMOS survey field to a nonconflicting right ascension. Our examination of alternative equatorial fields revealed a field near the VVDS $10 \mathrm{hr}$ field which in fact had slightly lower extinction $\left(E_{B-V} \simeq 0.02 \mathrm{mag}\right.$ ) and lower farinfrared cirrus backgrounds (Scoville et al. 2007) than the original $2 \mathrm{hr}$ VVDS/XMM-Newton Deep field. This field also has no extremely bright X-ray, UV, optical, or radio sources, unlike some other fields. This enables the COSMOS survey to efficiently observe the entire field to uniform sensitivity and to the optimum depth determined by the COSMOS science. However, since the $10 \mathrm{hr}$ field did not have as extensive prior observational coverage as the $2 \mathrm{hr}$ field, most of the ground-based optical/IR imaging would have to be done as part of the COSMOS survey.

In conclusion, the final field selected for COSMOS is a $1.4^{\circ} \times$ $1.4^{\circ}$ square, aligned east-west, north-south and centered at R.A. = $10^{\mathrm{h}} 00^{\mathrm{m}} 28.6^{\mathrm{s}}$, decl. $=+02^{\circ} 12^{\prime} 21.0^{\prime \prime}(\mathrm{J} 2000.0)$. It has low and exceptionally uniform optical extinction (less than $20 \%$ variation; see Sanders et al. 2007). Being equatorial, the field has somewhat higher far-infrared background than the very best fields such as the Lockman Hole (Scoville et al. 2007); however, this is clearly less detrimental to the overall survey than the penalty of very poor (or nonexistent) radio and millimeter/submillimeter coverage of higher declination fields by the VLA or ALMA. A summary of the HST observations is provided in Table 1; below, we briefly discuss each instrument in detail.

\subsection{ACS Observations and Processing}

Imaging with ACS in the F814W (I-band) filter is the primary COSMOS HST observation. The ACS WFC field of view (FOV) is $203^{\prime \prime} \times 203^{\prime \prime}$, covered by two CCD arrays separated by a gap of $4.5^{\prime \prime}$. The pixel size is $0.05^{\prime \prime}$. Nine of the allocated orbits were devoted to a test $3 \times 3$ pointing mosaic in the $\mathrm{F} 475 \mathrm{~W}$ ( $g$-band) filter at the center of the field in order to evaluate the need for full field coverage in a second filter. Thus, a total of 581 orbits/ pointings were devoted to imaging in the $I$-band filter. Within each orbit, four equal-length exposures of $507 \mathrm{~s}$ duration $(2028 \mathrm{~s}$ total) were obtained in a four-position dither pattern designed to shift bad pixels and to fill in the 90 pixel gap between the two ACS CCD arrays (see Koekemoer et al. 2007). Adjacent pointings in the mosaic were positioned with approximately $4 \%$ overlap in order to provide at least three-exposure coverage at the 
TABLE 1

HST OBSERVATIONS

\begin{tabular}{|c|c|c|c|c|c|c|}
\hline Instrument & Filter & Mode $^{a}$ & Orbits & $\begin{array}{c}\text { Exposure } \\
\text { (s) }\end{array}$ & Dates & Proposal ID \\
\hline ACS ............. & F814W ( $I$ band $)$ & $\mathrm{P}$ & 261 & 2028 & 2003 Oct $15-2004$ May 21 & 09822 (Сy 12) \\
\hline ACS & F475W ( $g$ band) & $\mathrm{P}$ & 9 & 2028 & 2003 Oct $15-2004$ May 21 & 09822 (Сy 12) \\
\hline ACS & $\mathrm{F} 814 \mathrm{~W}$ ( $I$ band $)$ & $\mathrm{P}$ & 320 & 2028 & 2004 Oct $15-2005$ May 21 & 10092 (Сy 13) \\
\hline NICMOS NIC3 .................... & F160W ( $H$ band $)$ & PP & 225 & 1536 & 2003 Oct $15-2004$ May 21 & 09999 (Су 12) \\
\hline NICMOS NIC3 .................... & F160W ( $H$ band $)$ & PP & 282 & 1536 & 2004 Oct $15-2005$ May 21 & 10337 (Су 13) \\
\hline WFPC2 & F300W ( $U$ band) & $\mathrm{CP}$ & 270 & 1600 & 2003 Oct $15-2004$ May 21 & 09822 (Сy 12) \\
\hline WFPC2 & F300W ( $U$ band) & $\mathrm{CP}$ & 149 & 1600 & 2004 Oct $15-2004$ Nov 21 & 10092 (Сy 13) \\
\hline 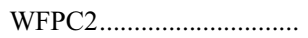 & $\mathrm{F} 450 \mathrm{~W}$ ( $B$ band) & $\mathrm{CP}$ & 171 & 1600 & 2004 Nov 21-2005 May 21 & 10092 (Су 13) \\
\hline
\end{tabular}

${ }^{\text {a }}$ Scheduling mode: (P) primary, (PP) pure-parallel, $(\mathrm{CP})$ coordinated-parallel.

TABLE 2

COSMOS HST SENSITIVITIES AND Resolution

\begin{tabular}{|c|c|c|c|c|c|c|}
\hline Instrument & Filter & $5 \sigma_{\mathrm{AB}}{ }^{\mathrm{a}}$ & $5 \sigma_{\mathrm{STmag}}$ & $5 \sigma_{\text {Vegamag }}$ & $\begin{array}{c}\text { Resolution (FWHM) } \\
\text { (arcsec) }\end{array}$ & $\begin{array}{l}\text { Pixel Scale } \\
\quad(\operatorname{arcsec})\end{array}$ \\
\hline . & F814W ( $I$ band $)$ & 28.6 & 29.5 & 28.2 & 0.09 & 0.05 \\
\hline ACS & F475W ( $g$ band) & 27.9 & 27.6 & 28.0 & 0.05 & 0.05 \\
\hline NICMOS NIC3 ..................... & F160W ( $H$ band) & 25.9 & 28.3 & 24.6 & 0.16 & 0.20 \\
\hline WFPC2 & F300W ( $U$ band $)$ & 24.8 & 26.1 & 24.8 & 0.10 & $0.1^{\mathrm{b}}$ \\
\hline WFPC $2 \ldots \ldots \ldots \ldots \ldots \ldots \ldots \ldots$ & F450W ( $B$ band) & 26.7 & 26.3 & 26.8 & 0.10 & $0.1^{\mathrm{b}}$ \\
\hline
\end{tabular}

a Sensitivities for optimally extracted point sources with a $\lambda^{-1}$ power-law spectrum for ACS and NICMOS. For WFPC2, an A0 star spectrum is assumed. For ACS and WFPC2 the flux is normalized to $V$, and for NICMOS the flux is normalized to $1.6 \mu \mathrm{m}$. The $V$-band normalization was adopted to be consistent with Table 3, where surveys in several bands are compared. Normalization of the flux to $I$ band reduces the ACS magnitudes by 1.4 mag (e.g., 28.6 to 27.2 ).

b Scale of $0.046^{\prime \prime}$ pixel $^{-1}$ for the PC.

TABLE 3

Relative Point Source Sensitivities

\begin{tabular}{|c|c|c|c|c|c|}
\hline Filter & Survey & $5 \sigma_{\mathrm{AB}}{ }^{\mathrm{a}}$ & Orbits & Vega - AB & STmag - AB \\
\hline \multirow[t]{2}{*}{ 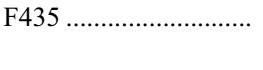 } & UDF & 29.94 & 56 & 0.11 & 0.52 \\
\hline & GOODS & 28.32 & 3 & ... & $\ldots$ \\
\hline \multirow[t]{2}{*}{ 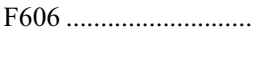 } & UDF & 30.84 & 56 & 0.09 & 0.17 \\
\hline & GOODS & 29.14 & 2.5 & $\ldots$ & ... \\
\hline \multirow[t]{2}{*}{ F775 } & UDF & 31.30 & 150 & -0.40 & 0.74 \\
\hline & GOODS & 29.04 & 2.5 & $\ldots$ & $\ldots$ \\
\hline F814 & COSMOS & 28.63 & 1 & -0.44 & 0.84 \\
\hline \multirow[t]{2}{*}{ 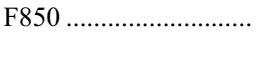 } & GOODS & 29.11 & 5 & -0.54 & 1.09 \\
\hline & UDF & 30.99 & 150 & $\ldots$ & $\ldots$ \\
\hline
\end{tabular}

Notes._GOODS: Giavalisco et al. (2004); UDF: Beckwith et al. (2006). To facilitate direct comparison of the different surveys, we have used the instrument exposure time calculators (ETCs) provided by STScI, rather than published sensitivities of the surveys (which were derived with differing assumptions).

a Sensitivities for optimally extracted point sources. For a source uniformly extended over $\sim 0.25^{\prime \prime}$ diameter, the limiting magnitudes are $\sim 1$ mag greater (assumes $2028 \mathrm{~s}$ per orbit for COSMOS and $2500 \mathrm{~s}$ per orbit for the other surveys, a $\lambda^{-1}$ power-law spectrum with normalization at $V$, four CR-splits per orbit for COSMOS, two CR-splits per orbit for GOODS and UDF, and no reddening). 


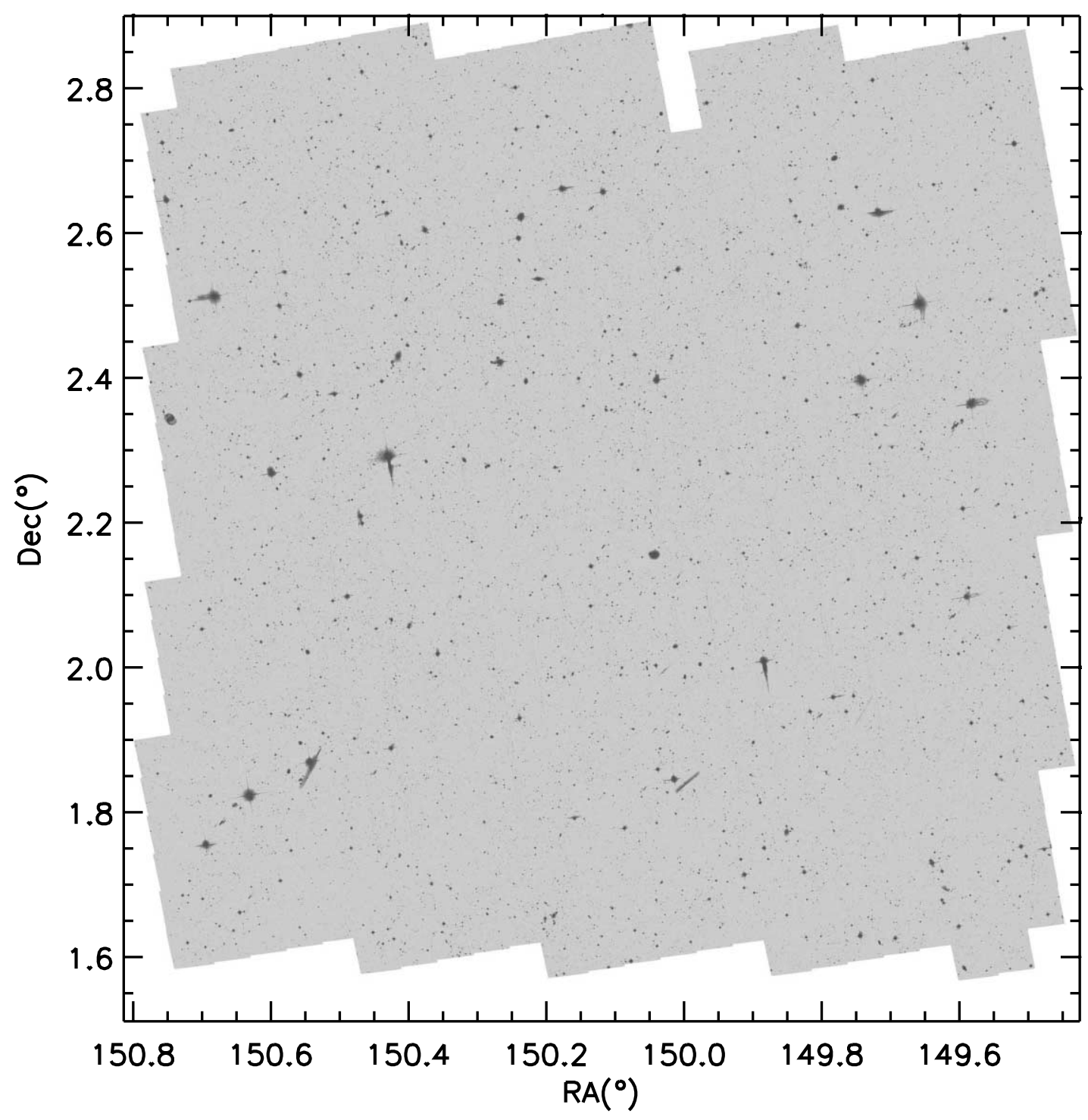

FIg. 1.-Layout of the ACS mosaic of $581 \mathrm{I}$-band pointings. The rectangle fully enclosing all the ACS imaging has lower left and upper right corners (R.A., decl. $[\mathrm{J} 2000.0])$ at $\left(150.7988^{\circ}, 1.5676^{\circ}\right)$ and $\left(149.4305^{\circ}, 2.8937^{\circ}\right)$. The WFPC2 $(3000$ and $4500 \AA)$ and NICMOS $(1.6 \mu \mathrm{m})$ images cover approximately $55 \%$ and $6 \%$ of the ACS area.

edge of each pointing and four-exposure coverage over approximately $95 \%$ of the survey area. This multiple exposure coverage with ACS provides excellent cosmic-ray rejection (Koekemoer et al. 2007).

The visibility windows for the COSMOS program were set such that two orientation angles opposed by approximately $180^{\circ}$ could be scheduled (P.A. $=290^{\circ} \pm 10^{\circ}$ and $110^{\circ} \pm 10^{\circ}$, corresponding to October 13-January 7 and March 2-May 21, respectively). (In the $H S T$ two-gyro mode, only the former is available.) Three of the pointings had large reflections or scattered light due to bright stars being on the edge of the ACS FOV. These three fields were later repeated with two exposures, placing the bright stars well within the ACS FOV. (They are included in the 581 orbit count mentioned above.)

A full description of the ACS data processing including drizzling, flux calibration, registration, and mosaicking is provided in Koekemoer et al. (2007). The registration was tied into groundbased CFHT I-band imaging (H. Aussel et al. 2007, in preparation) with the USNO-B1.0 reference frame offsets established from the COSMOS VLA survey (Schinnerer et al. 2007). The absolute registration of all ACS data in the COSMOS archive is accurate to approximately $0.05^{\prime \prime}-0.10^{\prime \prime}$ over the entire field (Koekemoer et al. 2007). The flux calibration of the ACS is tied into the standard STScI ACS calibration, accurate to better than $0.05 \%$ in absolute zero point. For the publicly released images with $0.05^{\prime \prime}$ pixels, the DRIZZLE parameters (Fruchter \& Hook 2002) were pixfrac $=0.8$ and a square kernel. For the images used for weaklensing analysis with $0.03^{\prime \prime}$ pixels, pixfrac $=0.8$, and a Gaussian kernel was used. (CTE effects on the faint source PSFs were reduced as described in Rhodes et al. [2007].) The final ACS mosaic image released to the public IRSA and MAST archives is sampled with $0.05^{\prime \prime}$ pixels. The measured FWHM of the PSF in the ACS $I$-band filter is $0.09^{\prime \prime}$. These individual images were also rotated to north up for the public release data. For the purpose of the weak-lensing analysis done by the COSMOS team, an internal release of the unrotated images, sampled to $0.03^{\prime \prime}$ pixels, was also generated to avoid rotating the original PSF and to reduce aliasing problems associated with resampling (Rhodes et al. 2007); for general morphological studies the rotated and the $0.05^{\prime \prime}$ images are entirely adequate. 


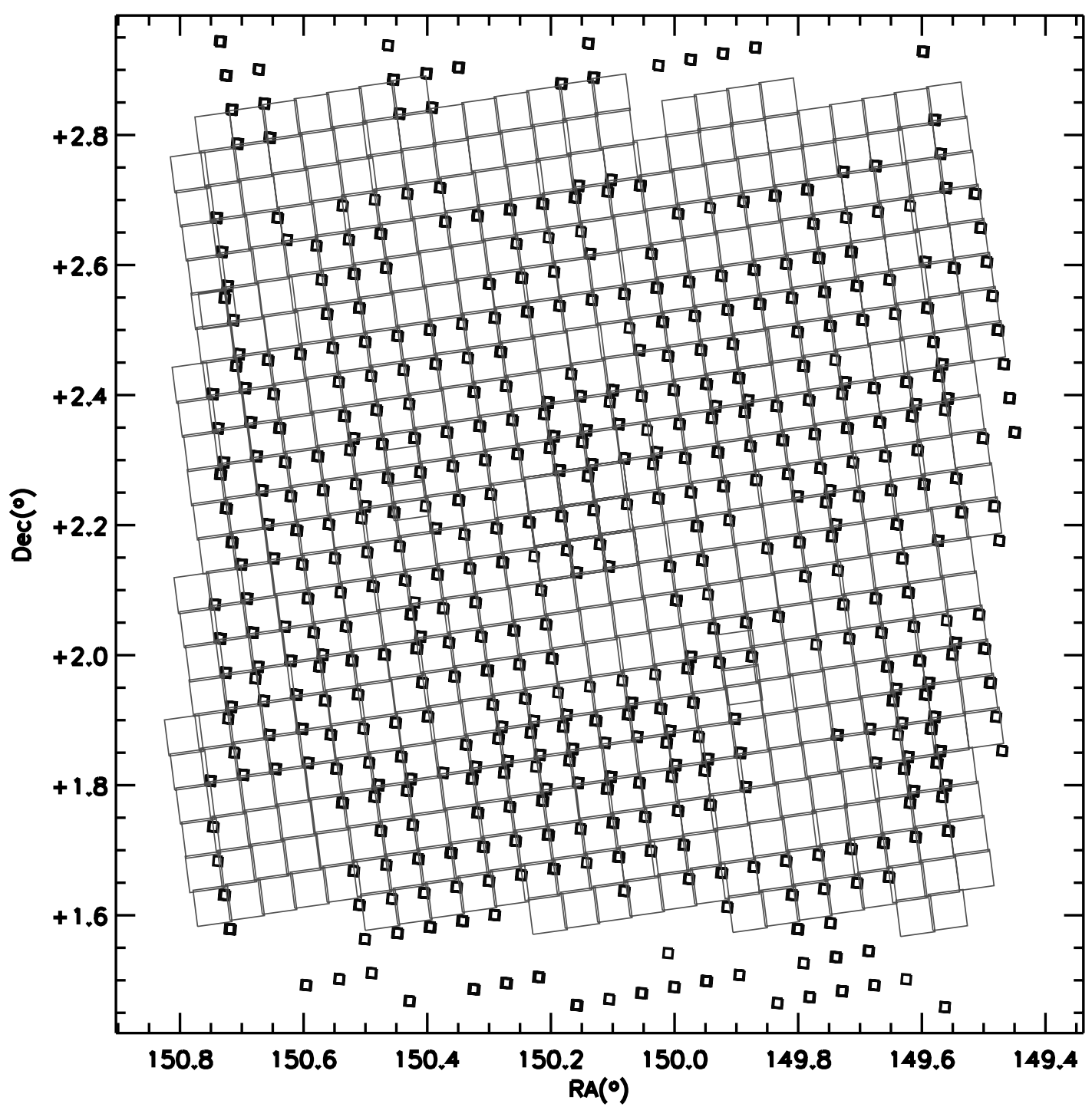

FIG. 2.-Layout of the NICMOS parallels superposed on the ACS pointings. The NICMOS $(1.6 \mu \mathrm{m})$ images cover approximately $6 \%$ of the ACS area.

\subsection{NICMOS and WFPC2 Parallel Observations}

In parallel with the ACS observations, imaging was obtained with NICMOS and WFPC2. WFPC2 was used in coordinatedparallel mode, implying that exposures were obtained with every ACS pointing. The WFPC2 FOV is $150^{\prime \prime}$, offset in position from the ACS field center by $5.8^{\prime}$. Since NICMOS cannot be used in periods of high particle flux such as during the South Atlantic Anomaly passages, the NICMOS observations were set up in pure-parallel mode so as not to impede scheduling of the primary ACS observations. Therefore, not every orbit has an associated NICMOS parallel observation. The NICMOS camera 3, used for COSMOS parallel observations, has a FOV of 50", and the field center is displaced $8.5^{\prime}$ from that of ACS.

For WFPC2, the filter used initially was F300W. However, inspection of the Cycle 12 data revealed a very low rate of object detection at $3000 \AA$; in the second half of Cycle 13, the WFPC2 filter was therefore changed to F450W. The NICMOS parallels used the F160W (1.6 $\mu \mathrm{m}, H$-band $)$ filter. There were four and three exposures per orbit for WFPC2 and NICMOS, respectively. The WFPC2 and NICMOS parallels cover approximately $55 \%$ $\left(1.07 \mathrm{deg}^{2}\right)$ and $6 \%\left(0.092 \mathrm{deg}^{2}, 330 \mathrm{arcmin}^{2}\right)$ of the COSMOS $1.8 \mathrm{deg}^{2}$ field imaged with ACS. The total areal coverage in NICMOS parallel imaging is probably the largest of any HST

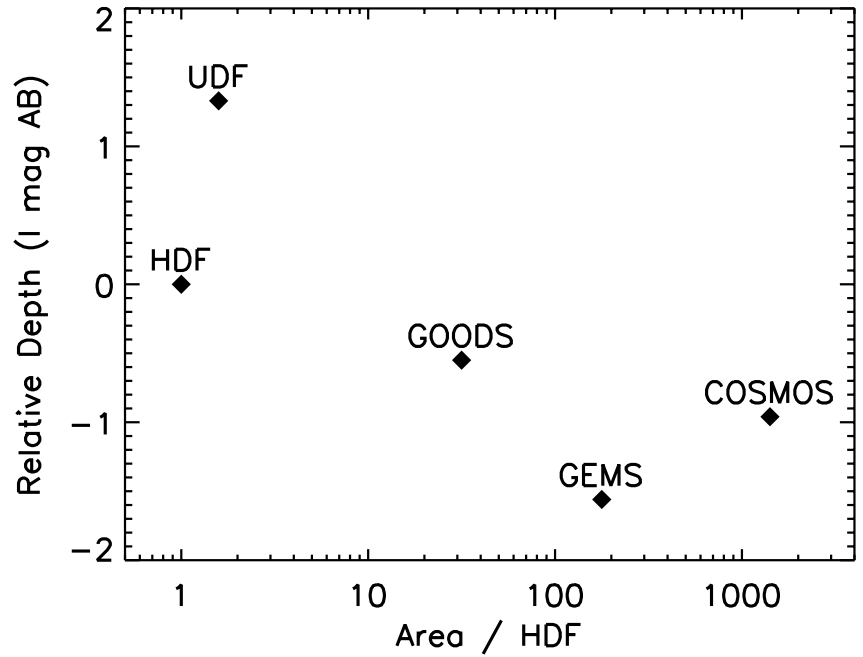

FIG. 3.-Relative field areas and sensitivities of major HST surveys at $\sim 8000 \AA$ compared to the original HDF survey. COSMOS has 9 times the area of GEMS (Rix et al. 2004; the next largest survey), with sensitivity just 1.4 times less than GOODS (Giavalisco et al. 2004) and in 20\% of the time due to the higher throughput of F814W vs. F850LP-GOODS. The relative sensitivities shown here were derived using the instrument ETCs (for an optimally extracted point source) in order to facilitate equivalent comparisons. 

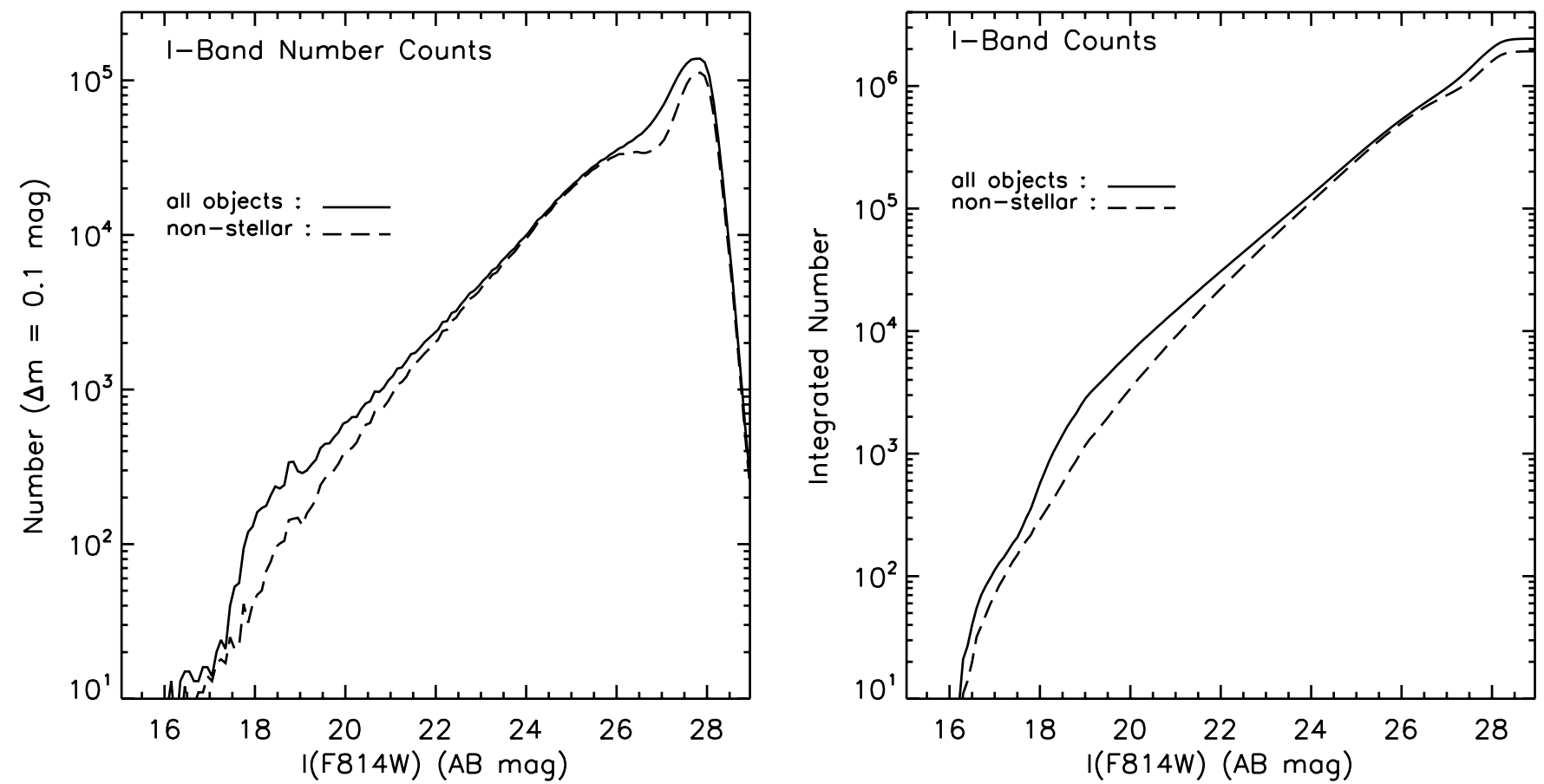

FIG. 4.- Source counts (differential, left; integral, right) for the COSMOS ACS $I$-band catalog (from SExtractor requiring signals $>0.4 \sigma$ in at least 4 adjacent pixels and a total isophotal signal-to-noise ratio $>1$ ). The number counts for automags are shown for all objects (solid line) and those with "stellarity" $<0.95$ (dashed line). The upturn at $I<27$ mag is the result of low signal-to-noise ratio spurious detections. These counts are not corrected for completeness.

project; although it is not contiguous, it provides enormous samples of objects. The details of reduction and calibration of the WFPC2 (done by S. Ewald) and NICMOS (done by J. Colbert) imaging are included in Koekemoer et al. (2007).

\section{SENSITIVITY, RESOLUTION, AND COVERAGE}

The sensitivities and resolutions for the COSMOS HST imaging are summarized in Table 2 and compared with those of other
HST surveys in Table 3. In order to facilitate direct comparison of the different surveys, we have used the instrument exposure time calculators (ETCs) provided by STScI, rather than the published sensitivities of the surveys, which were derived with differing assumptions. For ACS and WFPC2, the flux is normalized in the $V$ band; for NICMOS, the flux is normalized to $1.6 \mu \mathrm{m}$. (The $V$-band normalization was adopted to be consistent with Table 3, where the other HST surveys, which cover several bands,
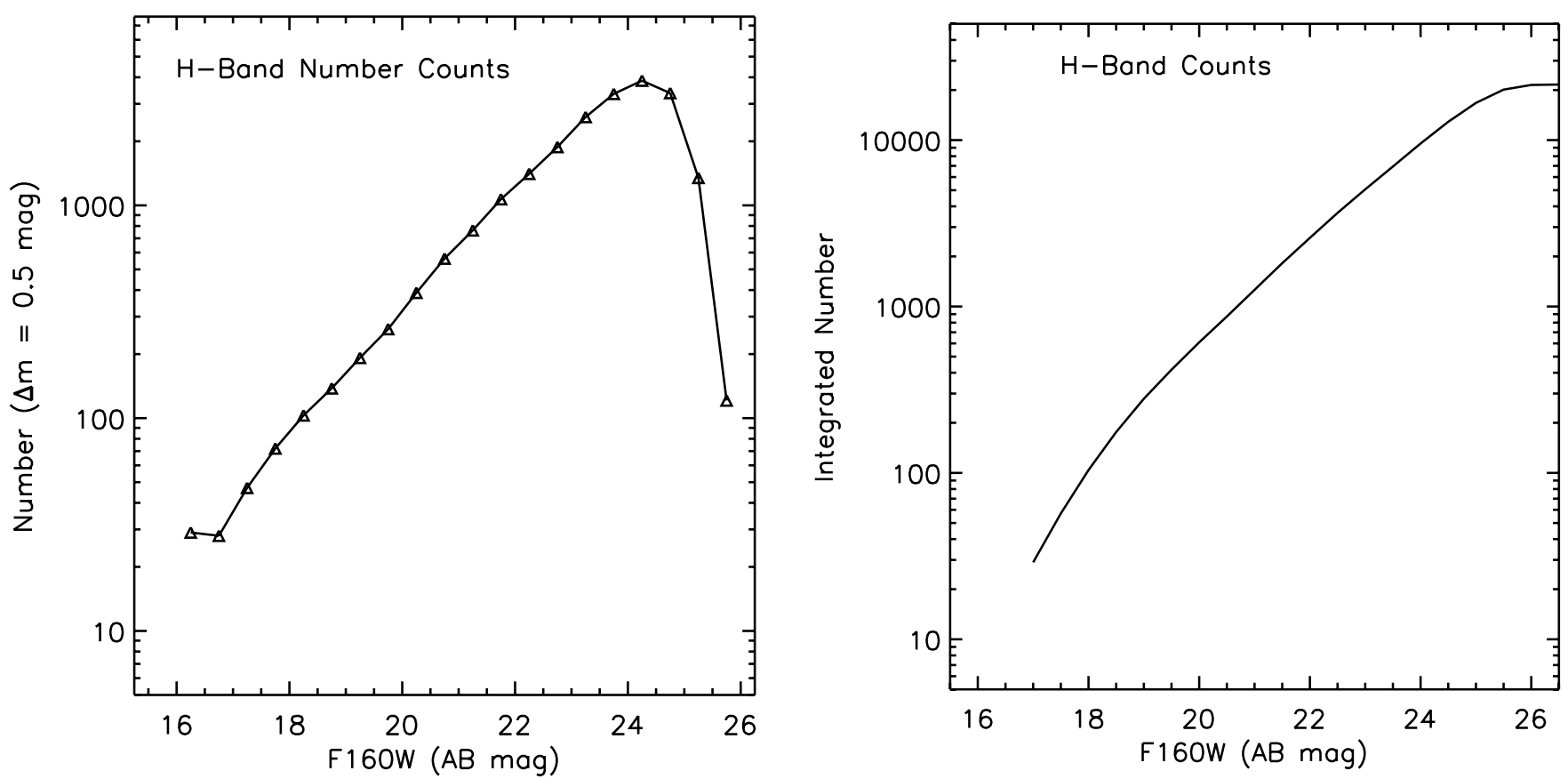

FIG. 5.-Source counts (differential, left; integral, right) for the COSMOS NICMOS $H$-band catalog (based on SExtractor measurements requiring a signal $>1.5 \sigma$ in 9 adjacent pixels). The number counts are for $0.5 \mathrm{mag}$ bins; the total area imaged in NICMOS is $0.099 \mathrm{deg}^{2}$. The total number of objects is 21,639 . 
TABLE 4

ACS Source Counts

\begin{tabular}{lcr}
\hline \hline $\begin{array}{c}I \text { Upper Limit } \\
(\mathrm{mag})\end{array}$ & All Objects & Nonstellar \\
\hline $25 \ldots \ldots \ldots$ & 288,657 & 266,039 \\
2 & 567,143 & 531,982 \\
& $1,029,007$ & 878,445 \\
& $2,166,291$ & $1,700,244$ \\
\hline
\end{tabular}

are summarized.) Normalization of the flux to $I$ band reduces the ACS magnitudes by $1.4 \mathrm{mag}$ (e.g., $28.6 \rightarrow 27.2 \mathrm{mag}$ ). The COSMOS ACS $I$-band coverage is shown in Figure 1. The rectangle bounding all the ACS imaging has lower left and upper right corners (R.A., decl. [J2000.0]) at $\left(150.7988^{\circ}, 1.5676^{\circ}\right)$ and $\left(149.4305^{\circ}, 2.8937^{\circ}\right)$. The positions observed in the NICMOS parallel observations are shown in Figure 2; the locations of the WFPC2 parallels are not shown in view of their low sensitivity (see below). In Figure 3 we show the relative areas and sensitivities of the major HST Treasury surveys.

\section{PHOTOMETRIC CATALOGS}

The primary reference catalogs for COSMOS have objects selected from both the ACS images and the very deep, multiband Subaru SCAM COSMOS imaging (Taniguchi et al. 2007). The SCAM data are of similar depth to the ACS imaging (approximately 0.8 mag deeper for sources larger than $1^{\prime \prime}$ in diameter, but for sources smaller than $\sim 0.3^{\prime \prime}$, the ACS data are more sensitive). The ground-based imaging (Capak et al. 2007) currently includes 18 filters including narrow- and intermediate-bandwidth filters. Catalogs were made from both the ACS and Subaru I-band and NICMOS $H$-band imaging. The ACS catalog comprises 646,470 objects detected at $>1 \sigma$; the NICMOS catalog has 21,639 objects ( $>1.5 \sigma$ or 9 adjacent pixels). For the ACS cata$\log$, SExtractor (Bertin \& Arnouts 1996) was used with the requirement of signals $>0.4 \sigma$ in at least 4 adjacent pixels and a total isophotal signal-to-noise ratio $>1$. The ACS catalog includes additional internal substructures within sources listed in the lower resolution, ground-based catalog. These catalogs and the derived photometric redshifts (Mobasher et al. 2007) are presented and described in detail elsewhere (Koekemoer et al. 2007; Capak et al. 2007a; Leauthaud et al. 2007).

\section{PUBLIC DATA RELEASE}

The COSMOS HST data are publicly available in staged releases (following calibration and validation) through the Web sites for IPAC/IRSA ${ }^{38}$ and STScI-MAST ${ }^{39}$ (The STScI pipeline processed images are of course also available in the STScI archive.) The COSMOS ACS imaging is in the form of separate drizzled images for each pointing, rotated and resampled to have north up with a pixel scale of $0.05^{\prime \prime}$. IRSA also supplies a cutout capability derived from the full-field mosaic (50 Gbytes). The cutouts can be made with any field center and size; and multiple cutouts are provided based on a user-supplied file containing

\footnotetext{
${ }^{38}$ See http://irsa.ipac.caltech.edu/data/COSMOS/.

39 See http://archive.stsci.edu.
}

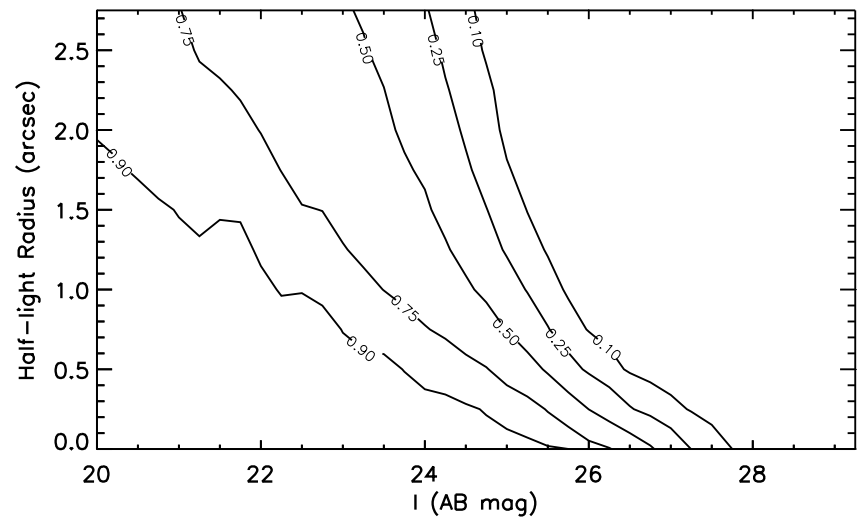

FIG. 6.-Completeness estimates for COSMOS ACS I-band imaging. Contours show the percentage of simulated sources (a 50/50 mix of exponential disks and spheroids) which were recovered by SExtractor as a function of source total magnitude and half-light radius.

source positions and field sizes. The SExtractor catalogs for ACS and NICMOS (see $\S 4$ ) are also available through IRSA.

\section{SOURCE COUNTS AND COMPLETENESS}

Figures 4 and 5 show the magnitude distributions of sources in the ACS and NICMOS source catalogs ( $(4)$. The ACS source counts are listed in Table 4. We have compared the ACS I-band source counts with those published by Ferguson et al. (2000) for the HDF. In the HDF, the derived $I$-band count at $I_{\mathrm{AB}}=25 \mathrm{mag}$ is $1.0 \times 10^{5}$ galaxies per square degree per $\Delta m=1 \mathrm{mag}$. For the COSMOS ACS catalog the count is $\sim 21,000$ for $1.8 \mathrm{deg}^{2}$ per $\Delta m=0.1 \mathrm{mag}$ (at $I_{\mathrm{AB}}=25 \mathrm{mag}$; see Fig. 4). The COSMOS counts are $\sim 16 \%$ higher than those in the HDFs, but given the fact that different SExtractor parameters were probably used in COSMOS and HDF, the agreement is quite acceptable. The $H$-band integrated number counts are equivalent to $3478,14,130$, and $41,300 \mathrm{deg}^{-2}$ per $0.5 \mathrm{mag}$ bin at $H=20,22$, and $24 \mathrm{mag}$ (AB). A recent compilation of previous surveys by Frith et al. (2006) has approximately $3300,15,000$, and $41,000 \mathrm{deg}^{-2}$ in the same bins (see dashed curve in their Fig. 1).

The completeness of the ACS catalog was determined using the standard technique of inserting false sources of specified halflight size and total flux (Giavalisco et al. 2004). Size and flux must both be explored, since the ability to detect sources depends on their surface brightness and hence their flux and size. The simulated galaxies were a 50/50 mix of exponential disks and $r^{1 / 4}$ spheroids (Giavalisco et al. 2004). Figure 6 shows contours for the percentage of the test galaxies recovered. For galaxies with half-light radii of $0.25^{\prime \prime}, 0.5^{\prime \prime}$, and $1^{\prime \prime}$, the completeness is $\sim 50 \%$ at $I_{\mathrm{AB}} \simeq 26.0,24.7$, and $24.5 \mathrm{mag}$.

\section{ANALYSIS ENABLED BY COSMOS ACS OBSERVATIONS}

The high-resolution ACS imaging is critical to the COSMOS survey, providing galaxy morphologies, multiplicities and merger rates out to $z \geq 2$, environmental density from dark matter maps at $z \leq 1$, and size and limited morphological information at redshifts out to $z=6$. The morphological parameters obtained from the ACS imaging include bulge/disk ratios, concentration, asymmetry, size, multiplicity, color, and clumpiness (see Cassata et al. 2007; Scarlatta et al. 2007; Capak et al. 2007b). The COSMOS $I$-band ACS images have sufficient depth and resolution to allow classical bulge-disk decomposition for $L^{*}$ galaxies at $z \leq 2$, 
while less detailed structural parameters such as concentration, asymmetry, clumpiness, and size can be measured for all galaxies down to the COSMOS spectroscopic survey limit (37,500 galaxies with $I_{\mathrm{AB}} \leq 25$; Lilly et al. 2007; Trump et al. 2007), out to $z \sim 5$. COSMOS ACS imaging has been crucial for the identification and analysis of galactic interactions and mergers (e.g., Kartalepe et al. 2007), processes which are central to the early evolution of galaxies.

For the purposes of weak-lensing analysis, approximately 87 galaxies arcmin $^{-2}$ were sufficiently resolved with ACS (cf. $\sim 30 \mathrm{arcmin}^{-2}$ from Subaru SCAM). Their median (mean) redshift is 1.02 (1.25), and their per-component rms shear is 0.309 . This permits mass reconstructions with an optimal resolution on the sky at scales of $\sim 100^{\prime \prime}$, and a redshift sensitivity that peaks between $z=0.2$ and 0.6 . These parameters enable detection of an $\sim 7 \times 10^{13} M_{\odot}$ cluster at $z=0.2$ with a $5 \sigma$ signal-to-noise ratio (Rhodes et al. 2007; Massey et al. 2007).
The HST COSMOS Treasury program was supported through NASA grant HST-GO-09822. We wish to thank Tony Roman, Denise Taylor, and David Soderblom for their assistance in the planning and scheduling of the extensive COSMOS observations. We gratefully acknowledge the contributions of the entire COSMOS collaboration, consisting of more than 70 scientists. More information on the COSMOS survey is available at http:// www.astro.caltech.edu/cosmos. It is a pleasure to acknowledge the excellent services provided by the NASA IPAC/IRSA staff (Anastasia Laity, Anastasia Alexov, Bruce Berriman, and John Good) in providing online archive and server capabilities for the COSMOS data sets. The COSMOS Science meeting in 2005 May was supported in part by the NSF through grant OISE-0456439. We thank Rob Kennicutt for suggestions on the manuscript.

Facilities: HST (ACS), HST (NICMOS), HST (WFPC2)
Beckwith, S. V. B., et al. 2006, AJ, 132, 1729

Bertin, E., \& Arnouts, S. 1996, A\&AS, 117, 393

Capak, P., et al. 2007a, ApJS, 172, 284 2007b, ApJS, 172, 99

Cassata, P., et al. 2007, ApJS, 172, 270

Davis, M., et al. 2007, ApJ, 660, 1

Ferguson, H., Dickinson, M., \& Williams, R. 2000, ARA\&A, 38, 667

Frith, W. J., Metcalfe, N., \& Shanks, T. 2006, MNRAS, 371, 1601

Fruchter, A. S., \& Hook, R. N. 2002, PASP, 114, 144

Giavalisco, M., et al. 2004, ApJ, 600, L93

Kartalepe, J., Sanders, D. S., \& Scoville, N. Z. 2007, ApJS, 172, 320

Koekemoer, A., et al. 2007, ApJS, 172, 196

Leauthaud, A., et al. 2007, ApJS, 172, 219

\section{REFERENCES}

Lilly, S. J., et al. 2007, ApJS, 172, 70

Massey, R. J., et al. 2007, ApJS, 172, 239

Mobasher, B., et al. 2007, ApJS, 172, 117

Rix, H.-W., et al. 2004, ApJS, 152, 163

Rhodes, J. R., et al. 2007, ApJS, 172, 203

Sanders, D. B.. et al. 2007, ApJS, 172, 86

Scarlata, C., et al. 2007, ApJS, 172, 494

Schinnerer, E. E., et al. 2007, ApJS, 172, 46

Scoville, N. Z., et al. 2007, ApJS, 172, 1

Taniguchi, Y., et al. 2007, ApJS, 172, 9

Trump, J. R., et al. 2007, ApJS, 172, 383

Williams, R. E., et al. 1996, AJ, 112, 1335

. 2000, AJ, 120, 2735 